\title{
LA GESTIÓN DE PROCESOS EN LOS PROYECTOS DE INVERSIÓN Y SU RELACIÓN CON LA PLANIFICACIÓN ESTRATÉGICA EN EL SECTOR ELÉCTRICO ECUATORIANO
}

\section{PROCESS MANAGEMENT IN INVESTMENT PROJECTS AND ITS RELATION TO STRATEGIC PLANNING IN THE ECUADORIAN ELECTRICITY SECTOR}

\author{
Diana Calderón Cabello, Mgtr. \\ Universidad Tecnológica ECOTEC, Samborondón, Ecuador. \\ dianacalderon85@hotmail.com \\ Guillermo Granja Cañizares, Mgtr. \\ https://orcid.org/0000-0002-7619-3350 \\ Universidad Tecnológica ECOTEC, Samborondón, Ecuador. \\ ggranja@ecotec.edu.ec
}

\section{ARTÍCULO DE INVESTIGACIÓN}

Recibido: 26 de mayo de 2021

Aceptado: 8 de octubre de 2021

\section{RESUMEN}

Este trabajo de investigación tiene como objetivo analizar la Gestión de procesos en los Proyectos de Inversión y su relación con la planificación estratégica en el sector eléctrico ecuatoriano. Para poder realizarlo se utilizó un estudio de tipo descriptivo, con un enfoque mixto, cuyos instrumentos de medición fueron principalmente las series de datos, y los indicadores de gestión asociados a los proyectos, teniéndose como variables al Plan de Gestión de Proyectos y la Planificación Estratégica. Los principales hallazgos fueron que no existe una planificación estratégica adecuada que permita soportar un Plan de Dirección de Proyectos, desde la concepción de una iniciativa, la cual posterior a su aprobación se considerará un proyecto, así como también la ejecución en general de los mismos; además en lo que concierne a estos, los alcances de los proyectos tienen factores críticos como presupuesto, especificaciones o características propias, grupo de interés, procesos y procedimientos propios, tiempos, entre otros. Además, cuando estos son ejecutados, la comunicación entre los involucrados y las políticas establecidas en cada una de las áreas inmersas en la ejecución, en ocasiones difieren afectando a los tiempos establecidos dentro del plazo programado para su realización o finalización.

Palabras claves: planificación estratégica, proyectos de inversión, sector público. 


\section{ABSTRACT}

The objective of this research work is to analyze the process management in investment projects and its relationship with strategic planning in the Ecuadorian electricity sector. In order to do so, a descriptive study was used, with a mixed approach, whose measurement instruments were mainly data series and management indicators associated to the projects, having as variables the Project Management Plan and Strategic Planning. The main findings were that there is no adequate strategic planning to support a Project Management Plan, from the conception of an initiative, which after its approval will be considered a project, as well as its execution in general; in addition, regarding these, the project scopes have critical factors such as budget, specifications or characteristics, stakeholders, processes and procedures, time, among others. In addition, when these are executed, the communication between those involved and the policies established in each of the areas involved in the execution, sometimes differ, affecting the times established within the programmed term for its realization or completion.

Keywords: strategic planning, investment projects, public sector

\section{INTRODUCCIÓN}

La gestión y ejecución de proyectos dentro del sector público o administraciones gubernamentales se encuentra muy limitada. Existe una gran cantidad de proyectos a gestionar y ejecutar, cuyos procesos inmersos o que generan valor, presentan una serie de limitantes que impactan de forma negativa en la consecución de sus objetivos. Dentro del sector público, el éxito del proyecto no representa un beneficio económico, sino más bien ofrece beneficios sociales, de tal forma que la manera de medir el éxito de los mismos es con base en la satisfacción que da a sus beneficiarios, en este caso a la ciudadanía, y si estos no son llevados a cabo dentro de los diversos compromisos que se generan a la comunidad, significará un punto en contra sobre la mejora de calidad de vida a la misma. Para aportar a una mejora en lo que respecta a la calidad de vida de una sociedad, es necesario efectuar una correcta gestión de proyectos de inversión en el ámbito social.

De acuerdo a lo manifestado por (Juan José Miranda Miranda, 2002) :El desarrollo de una sociedad, sin lugar a duda, guarda relación con lo que el sector público invierte en su comunidad, por lo que se determina que a mayor inversión, se obtienen mayores niveles de crecimiento social, así que considera imperante contar con herramientas idóneas que permitan identificar los proyectos que aporten al crecimiento de la economía y que garanticen también crecimiento y bienestar social. 
Sobre la necesidad de gestionar proyectos, se hace énfasis en que: "Los proyectos constituyen el núcleo de muchas iniciativas estratégicas. Es una forma de desplegar las estrategias y alcanzar sus objetivos establecidos" (PMI Madrid Spain Chapter, 2018).

Sobre las problemáticas presentadas durante la ejecución y gestión de proyectos de inversión, se nombran algunos elementos que, de manera dinámica, impactan de diversas formas a los mismos, como por ejemplo: involucrados o también llamados stakeholders, lo cual se refiere a todas las personas que influyen directa e indirectamente en un proyecto, riesgos, factores que pueden afectar negativamente si es que estos no son identificados y tratados oportunamente, y al proceso de integración, referente a la relación de todas las áreas de conocimiento de un proyecto, como por ejemplo alcance, tiempo, costo, calidad, recursos humanos, comunicación, riesgos y adquisiciones) (Revista Ciencias Estratégicas, 2016).

Ante lo expuesto, se presenta la siguiente problemática: ¿Los proyectos de inversión en el Sector Público cuentan con una adecuada gestión de los procesos y una correcta planificación estratégica que permita que estos cumplan con los objetivos estratégicos de las instituciones gubernamentales?

La ejecución eficiente de los proyectos permite cumplir los objetivos de la organización, inmersos en el Plan Estratégico Institucional, por lo que es imperante tener un control adecuado de tal forma que estos proyectos se ejecuten conforme a lo planificado y logren generar valor a la misma, por lo que una correcta planificación estratégica y de procesos logra que los proyectos de inversión se puedan ejecutar adecuadamente, para ello se plantea como objetivo general: analizar la Planificación Estratégica en lo que concierne a los procesos de gestión relacionados a la ejecución de los proyectos de inversión pública.

El Project Management Institute manifiesta que: "Un proyecto es un esfuerzo temporal que se lleva a cabo para crear un producto, servicio o resultado único" (Project Management Institute, 2017). Por otro lado, con respecto a la administración estratégica, se indica que "se refiere a un proceso de evaluación sistemática de un negocio y define los objetivos a largo plazo, identifica metas y objetivos, desarrolla estrategias para alcanzar estos y localiza recursos para realizarlos" (ESAN, 2016). De igual forma es preciso indicar que un proceso es un flujo de actos realizados en orden, enfocados hacia un fin (Concepto Definición, 2019). 
Los aportes metodológicos están fundamentados en el estado del Arte, conforme lo siguiente: Según Efraín Amaya Vanegas, en su tesis denominada Diseño de la Oficina de Proyectos (PMO) para Oleoducto Central S.A., indica que el desarrollo de proyectos en empresas del sector energético es uno de los mecanismos que se emplean para alcanzar las metas establecidas, así como también su éxito determina el logro de sus objetivos en términos de crecimiento, rentabilidad y sostenibilidad (Vanegas).

Con respecto a la administración de proyectos, Jimmy Ochoa Coronel, en su implementación de una metodología para la gestión de proyectos en una empresa eléctrica, indica que se consideran necesarias las implementaciones de procesos y procedimientos relacionados a la gestión de programas o conjunto de proyectos, ya que esto a su vez aporta a la optimización de plazos, reducción o ahorro en las inversiones planificadas inicialmente y esto sin duda alguna refleja una optimización en el plan anual de inversiones, incrementando sus índices en las ejecuciones presupuestarias anuales en las instituciones públicas (Ochoa, 2018).

El aporte práctico de esta investigación, se enfoca en los factores internos y externos y los cuales son limitaciones, ya que sin duda alguna impactan en los proyectos de inversión, contribuyendo a que estos no logren culminarse de forma exitosa y que a su vez no se liquiden o terminen dentro de sus parámetros iniciales, es decir que no logran cumplir con su tiempo, costo, alcance y calidad con los que fueron planificados y aprobados por los niveles superiores jerárquicos.

Debido a que es necesario gestionar y ejecutar proyectos como un medio para que una institución logre cumplir con los objetivos estratégicos, se considera necesario conocer que una incorrecta planificación de su desarrollo y el no tener claro cuál es el alcance y hacia donde se pretender llegar, conlleva a un sin número de reprocesos, incremento en los recursos y de tiempo, afectando a las comunidades consideradas usuarios finales, ya que las instituciones públicas o gubernamentales desarrollan proyectos de índole social, siendo el fracaso su único resultado.

Como resultado de una correcta gestión de proyectos, se logran palpar los beneficios, como por ejemplo cumplir con la demanda que la sociedad genera, convertir todos aquellos errores e inconvenientes como lecciones aprendidas y aplicarlos en nuevos proyectos desarrolladas como mejora continua, madurar la gestión de comunicación entre los diversos interesados, y el asegurar la calidad en cada una de las entregas finales de sus obras o proyectos. 


\section{REVISIÓN DE LA LITERATURA}

\section{Teorías de la administración estratégica}

La Administración Estratégica es considerada como una herramienta que a su vez va a permitir a los altos niveles el facilitar un proceso de mejora continua en cada una de las organizaciones. Es un proceso que permite diseñar, evaluar, definir y llevar a cabo cada una de las estrategias, permitiendo incrementar su nivel de competencia y éxito, sea este a corto, mediano o largo plazo, permitiendo también realizar un análisis situacional o toma de decisiones que sin duda alguna va a permitir desarrollarse de forma más eficiente (Zacarías Torres Hernández, 2014).

\section{Teorías de la gestión de procesos}

Un proceso significa un conjunto de actividades relacionadas y que trabajan de forma interactiva entre sí y que, a través de un procedimiento, convierten componentes de entrada en un producto o resultado final. La gestión de procesos es considerada como uno de los principales principios de la gestión de calidad, cuando todos los recursos empleados y las actividades se gestionan de forma integrada, permitiendo alcanzar su nivel de eficiencia máxima (Asociación española para la calidad, 2019).

Es necesario definir indicadores, cuyos resultados permitirán identificar de manera cualitativa y cuantitativa la situación actual, tomar medidas preventivas cuando se está próximo a incurrir en desviaciones sobre lo planificado, o correctivas cuando estas desviaciones ya se han presentado, para lo cual se debe también identificar líneas bases que, a través de un proceso de control de cambios, permita introducir estas mejoras para poder suplir las necesidades presentadas.

Una de las mayores ventajas de la gestión de procesos es la calidad, que se mide como el grado con el que se cumple los requisitos de un cliente, permitiendo reducir costos en la operatividad de una organización. Es considerada como una condición que debe tener todo servicio para poder obtener un mayor rendimiento en cada una de sus actividades y que aporte a una mayor durabilidad de la organización, creando confianza en cada uno de sus clientes y marcando fronteras para un mejor futuro de la empresa (IDDEO Consulting).

\section{Teorías de la gestión de proyectos}

Un proyecto es un esfuerzo temporal realizado para crear un producto o un servicio único, a través de una serie de acciones que se llevan a cabo. Todo proyecto debe cumplir con un plazo, es decir que cuenta con una fecha de inicio y una fecha de fin, un alcance que es una descripción de que es lo que se pretende realizar y hacia donde se quiere llegar, y por último un presupuesto, siendo este el recurso económico con el que se dispone para ejecutar el 
mismo. Un proyecto permite crear resultados duraderos y es el medio con el cual una organización lleva a cabo un cambio o una mejora. (Project Management Institute, 2017).

Un proyecto es creado para poder efectuar una planificación estratégica, aportando al incremento del nivel de excelencia, y logrando que la organización entregue productos y servicios de calidad. El Project Management Institute (PMI), indica que los proyectos impulsan cambios para poder generar valor dentro de las organizaciones (Project Management Institute, 2017).

La gestión de proyectos corresponde al uso del conocimiento, técnicas y habilidades para que estos sean ejecutados de la forma más idónea, es una competencia de índole estratégico que permite vincular los resultados del mismo con los objetivos estratégicos o metas establecidas por una organización (Edinburgh Business School, 2014).

\section{Plan de dirección de proyectos}

El plan de dirección de proyectos es un documento o grupo de documentos que se encuentran aprobados de manera formal, y que en conjunto sirve de guía para dirigir la planificación, ejecución, monitoreo y control, y cierre de un proyecto, grupo de procesos que conforman la gestión de un proyecto como tal.

Es importante mencionar que un plan de dirección de proyectos abarca todos los procesos que serán empleados por un proyecto, su interacción y dependencia desde su planificación hasta su culminación, así como también las herramientas y técnicas a emplear, descripción de cómo se efectuarán los cambios a las líneas bases (presupuesto, tiempo y alcance establecidos desde el inicio), quienes aprobarán los mismos y como se manejará el monitoreo y control de estos.

Todo plan de dirección de proyectos abarca el ciclo de vida del mismo, relacionado con sus grupos de procesos y sus 10 áreas de conocimiento (integración, alcance, cronograma, costos, calidad, recursos, comunicación, riesgo, adquisiciones e interesados) (Project Management Institute, 2017). 


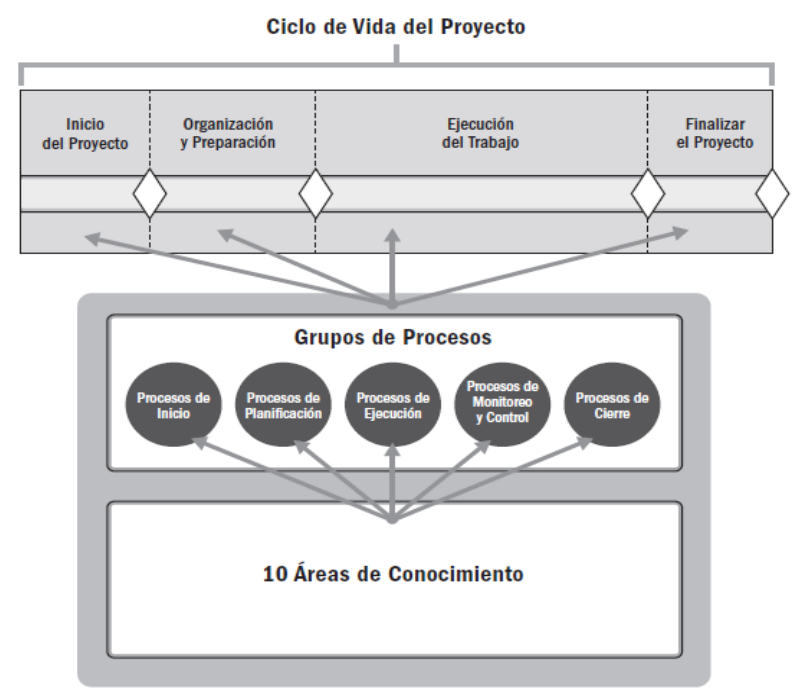

Figura 1 . Ciclo de vida de un proyecto

Fuente: (Project Management Institute, 2017).

De esta revisión de la literatura se considera importante tener en claro las metas y los objetivos establecidos para la elaboración de este trabajo, lo cual servirá de aporte para aquellas organizaciones que necesiten metodologías en proyectos, o a su vez los gestionen con buenas prácticas o metodologías establecidas. Es importante que, antes de comprometer recursos, se definan claramente las metas y objetivos que se pretenden alcanzar, identificando todos los factores que pueden afectar de forma positiva y negativa, los recursos indispensables y los procesos e involucrados, internos o externos, y que a través de los cuales se van a desarrollar todas las acciones.

Tener la experiencia, habilidades y conocimientos es la clave principal que permite a todo Gestor de Proyectos o PMP (Project Management Professional) liderar a un equipo de trabajo, que se encuentre comprometido con su rol asignado y que a su vez ese compromiso se refleje en las tareas encomendadas y resultados obtenidos durante la ejecución de un proyecto.

\section{MATERIALES Y MÉTODOS}

Dentro de la metodología de la investigación debe destacarse lo siguiente y que será aplicado en función al objeto de estudio: El tipo de investigación es descriptiva, permite analizar de forma exacta las actividades, objetos, procesos y personas involucradas en la gestión de proyectos de inversión que son llevados en las instituciones gubernamentales. El enfoque de investigación es cuantitativo, se analizará de forma crítica las variables inmersas en una ejecución de proyectos, sus procesos como son el de planificación, ejecución y liquidación, inclusive las áreas organizacionales que se encuentran involucradas en la gestión de los mismos. También se analizarán datos estadísticos de los tiempos de gestión y los recursos necesarios para su ejecución, factores importantes que impactan de forma significativa en la ejecución de diversos proyectos. 
El método de investigación es analítico sintético porque a partir del análisis de las etapas de un proyecto y las diversas situaciones presentadas en cada una de ellas, se pueden analizar las causas por las cuales los proyectos presentan inconvenientes, y que efectos pueden generarse evitando que culminen dentro de lo planificado o con el éxito deseado, satisfaciendo las necesidades de comunidades.

Las técnicas de investigación que se emplearán son: Revisión documental, ya que una institución gubernamental debe cumplir con lo estipulado en políticas o lineamientos establecidos o impartidos por las diferentes entidades de control, como en el caso del sector eléctrico tenemos la Agencia de Regulación y Control de Electricidad- ARCONEL, Ministerio de Energía y Recursos Naturales No Renovables -MERNNR o el Servicio Nacional de Contratación Pública - SERCOP, revisión bibliográfica y la observación directa, ya que se analizarán las prácticas que son empleadas en el ámbito administrativo y operacional.

Los instrumentos de medición serán serie de datos, basadas en los datos históricos sobre la culminación de proyectos, cuántos resultaron exitosos y cuántos no, o si estos culminaron dentro del plazo y con el presupuesto aprobado, tomando como referencia una industria en particular dentro del sector público, como ejemplo la comercialización y distribución de energía. Finalmente, las variables de investigación son: Dependiente: Sector Eléctrico, e Independiente: Planificación estratégica y proyectos de inversión, en donde se definen los objetivos hacia los cuales apunta la ejecución de los proyectos y sobre qué indicadores estos son medidos, derivados de la ejecución de estos.

Tabla 1

\section{Metodología Aplicada}

\begin{tabular}{|c|c|c|c|c|c|c|}
\hline $\begin{array}{l}\text { Variables de } \\
\text { Investigación }\end{array}$ & $\begin{array}{l}\text { Indicadores } \\
\text { de Variable }\end{array}$ & $\begin{array}{c}\text { Tipo de } \\
\text { Investigación }\end{array}$ & $\begin{array}{c}\text { Enfoque } \\
\text { Investigación }\end{array}$ & $\begin{array}{l}\text { Método de } \\
\text { Investigación }\end{array}$ & $\begin{array}{l}\text { Técnicas de } \\
\text { Investigación }\end{array}$ & $\begin{array}{l}\text { Instrumentos } \\
\text { de Medición }\end{array}$ \\
\hline $\begin{array}{l}\text { Dependiente } \\
\text { Sector } \\
\text { Eléctrico }\end{array}$ & $\begin{array}{l}\text { Análisis } \\
\text { Sectorial } \\
\text { Análisis } \\
\text { Competencia }\end{array}$ & & & & $\begin{array}{l}\text { Revisión } \\
\text { Documental }\end{array}$ & \\
\hline $\begin{array}{l}\text { Independiente } \\
\text { Planificación } \\
\text { Estratégica }\end{array}$ & $\begin{array}{l}\text { Objetivo } \\
\text { Estrategia } \\
\text { Táctica }\end{array}$ & Descriptiva & Cuantitativo & $\begin{array}{l}\text { Analítico } \\
\text { Sintético }\end{array}$ & $\begin{array}{l}\text { Revisión } \\
\text { Bibliográfica } \\
\text { Observación } \\
\text { Directa }\end{array}$ & $\begin{array}{l}\text { Series } \\
\text { Datos }\end{array}$ \\
\hline $\begin{array}{l}\text { Proyectos de } \\
\text { Inversión }\end{array}$ & $\begin{array}{l}\text { Monto } \\
\text { Rentabilidad } \\
\text { Impacto } \\
\text { social }\end{array}$ & & & & & \\
\hline
\end{tabular}

Fuente: Elaboración propia 


\section{RESULTADOS}

\section{Análisis sectorial}

Tomando como punto de partida el sector eléctrico y su distribución, este en los últimos 10 años ha estado encaminado a abastecer de servicio, dando impulso al desarrollo de los recursos energéticos actuales como también dando priorización al uso de recursos renovables, ejecutando obras que permiten garantizar el abastecimiento del servicio eléctrico de forma adecuada. Así también, se ha fortalecido la infraestructura institucional y se han realizado acciones para mejorar las gestiones administrativas de las empresas eléctricas, permitiendo que la expansión del servicio incremente y mejore (Ministerio de Energía y Recursos Naturales No Renovables, 2019).

En lo que respecta a la gestión comercial, se han mejorado los indicadores de facturación y recaudación de la energía eléctrica, recaudación de la cual se obtienen los recursos para la operatividad y mantenimiento de toda la red eléctrica, regenerando un servicio con sostenibilidad en el tiempo. Con base en estadísticas presentadas por ARCONEL, la recaudación desde el año 2008 al 2018 ha incrementado de $93.69 \%$ a 98.43\%.

\section{Análisis competencia}

Una de las Empresas Eléctricas más grandes del país es la Corporación Nacional de Electricidad, CNEL EP, con una cobertura del $45.05 \%$ del área total nacional, y cuyos abonados se encuentran agrupados en 4 grupos de consumo: residencial, industrial, comercial y alumbrado público. Esta corporación registra el $60.83 \%$ de la facturación a nivel nacional y su facturación representa el $61.78 \%$ de la totalidad del país.

La función de distribución de energía en el país está conformada por 9 empresas eléctricas más, encargadas de expandir el servicio eléctrico en el territorio nacional restante: EE. Sur, EE. Norte, EE. Quito, EE. Ambato, EE. Centro Sur, EE. Riobamba, EE. Cotopaxi, EE. Azogues y EE. Galápagos.

Del total de la facturación a Nivel Nacional, CNEL registra el $60.83 \%$ en comparación con las demás distribuidoras, conforme estadísticas efectuadas por la Agencia de Regulación y Control de Electricidad a diciembre de 2018, y el 61.78\% de sus ingresos en el año 2018.

La recaudación a nivel nacional fue de: 409.88 millones (23.38\% industrial), 654.81 millones (37.34\% residencial), 394.49 millones (22.50\% comercial) y 294.28 millones (16.78\% alumbrado público y otros).

En el sistema de distribución para el año 2018, la energía fue de 23.745,35 GWh, de las cuales 2.705,29 GWh corresponden a pérdidas, representando el $11.39 \%$ a nivel nacional (Sistema Único de Información Estadística del Sector Eléctrico Ecuatoriano). 
Debido que las empresas distribuidoras compran energía a las empresas generadoras y estas cobran en el punto de entrega todo lo que las distribuidoras consumen, en esta facturación se incluye el consumo por el servicio vendido al cliente y todo aquel consumo que ese genera por las pérdidas técnicas y no técnicas, representando estas últimas valores que deben ser cancelados por parte de las distribuidoras sin que estos correspondan a ventas del servicio, afectando a la disponibilidad de recursos económicos para la postulación y ejecución de nuevos proyectos a beneficio de la sociedad, o a su vez para invertir en la operación y mantenimiento de las redes eléctricas.

Con el objetivo de disminuir las pérdidas técnicas, se llevan a cabo proyectos comerciales para poder efectuar el balanceo de las cargas y así evitar la sobredimensión en los equipos eléctricos o disipación de energía dimensionando la misma; en lo que respecta a las pérdidas no técnicas las cuales corresponden al hurto (robo) del servicio eléctrico, se llevan a cabo proyectos para el cambio de redes desnudas a pre ensambladas, evitando de esta manera que los usuarios finales puedan conectarse de forma directa, así también se efectúan cambios de acometidas y medidores a equipos inteligentes, que permiten detectar cuando el cliente manipula el medidor y alerta posibles hurtos, contribuyendo a bajar los niveles de pérdidas.

\section{Planificación estratégica}

Guardando relación con las políticas del gobierno nacional, las empresas públicas definen objetivos estratégicos basados en cuatro perspectivas: clientes, sostenibilidad financiera, productividad y eficiencia, y aprendizaje y desarrollo.

Una Oficina de Proyectos o PMO, dentro de una institución gubernamental, es la encargada de otorgar buenas prácticas o metodologías de gestión de proyectos, así como también llevar el control y monitoreo de los mismos, sean estos financiados con presupuesto general del estado o con recursos derivados de su recaudación, y la gestión de estos debe aportar al cumplimiento de los objetivos estratégicos definidos en su plan.

Es importante considerar que dentro del sector público existen Oficinas de Proyectos funcionales, las cuales no son responsables de ejecutar directamente los proyectos, siendo estos efectuados directamente por las áreas operativas quienes tiene sus roles y funciones ya delegadas y asignadas conforme su cargo lo describe, como por ejemplo las áreas fuertes del negocio sea la Gerencia de Distribución o Gerencia Comercial, sin embargo, la Oficina de Proyectos gestiona y coordina de forma interna y externa todas las actividades a realizarse dentro de su ejecución, sea en su etapa precontractual, contractual y liquidación, en coordinación con las diversas áreas de apoyo, sean estas financiero, legal, adquisiciones, etc. 
Considerando el ejemplo de una empresa distribuidora de energía, se pueden analizar objetivos estratégicos como por ejemplo: Incrementar la eficiencia financiera en la operación y los proyectos, Incrementar los niveles de eficiencia de distribución eléctrica y alumbrado público, e Incrementar la eficiencia en gestión de ingresos, todos vinculados ya que los proyectos se miden a nivel físico (operativo o de ejecución) y a nivel financiero (ejecución financiera o avance presupuestario), los cuales deben realizarse dentro de los plazos establecidos por los entes de control.

Cabe mencionar que los proyectos de inversión hacen referencia a actividades temporales y únicas, que cuentan con un plazo determinado, por tal motivo las estrategias vinculadas a los objetivos identificados corresponden a:

- Minimizar las pérdidas no técnicas.

- Fortalecer la gestión financiera de costos de proyectos.

- Expandir la cobertura de servicio de distribución eléctrica y alumbrado público.

- Incrementar la eficiencia de la red y calidad del producto.

Toda estrategia cuenta con sus respectivos indicadores, permitiendo conocer en qué medida se cumplen los objetivos estratégicos o si en estos existen desviaciones que permitan alertar a la toma de decisiones preventivas o correctivas, así como también evaluar el desempeño o desenvolvimiento que está teniendo la organización en la gestión de sus proyectos, que deben satisfacer las necesidades de las comunidades, ya que esto también se pacta en los diferentes compromisos ministeriales o presidenciales.

Las tácticas empleadas para cada estrategia se definen de la siguiente manera:

- Ejecución de proyectos de cambio de redes desnudas a pre ensambladas, que permitirán medir el porcentaje de pérdidas no técnicas.

- Llevar a cabo proyectos de telemetría (lectura inteligente).

- Llevar un control de la ejecución presupuestaria, sea por proyecto y por programa (conjunto de proyectos).

- Incrementar la cobertura, llevando a cabo proyectos de expansión del servicio eléctrico.

- Ejecutar proyectos que permitan incrementar el alumbrado público.

- Realizar planes de mantenimiento para mejorar la calidad del servicio.

- Realizar adquisiciones de equipos de calidad que permitan dotar de un mejor servicio, mejorando los parámetros técnicos: nivel de voltaje, curva de voltaje, balanceos, etc. 
Se considera necesario que, de forma periódica, sea mensual, trimestral o semestral, se evalúen los cumplimientos de indicadores conforme a la ejecución de las diferentes estrategias y tácticas definidas.

\section{Proyectos de inversión de la gerencia de planificación}

\section{Monto}

En la actualidad, un plan anual de inversión de una empresa distribuidora en el año 2020 está conformado por aproximadamente 1800 proyectos de inversión por un monto aproximado de USD \$270 millones de dólares y, con base en estudios efectuados, la ejecución presupuestaria de este tipo de organizaciones llegó a un promedio del 4\%, y que en comparación con las metas establecidas por los entes reguladores, obtuvo una diferencia de un $36 \%$ para el primer trimestre ( $40 \%$ meta establecida).

Es importante mencionar que la ejecución presupuestaria desde el año 2018 fue disminuyendo hacia el 2020, esto refleja que la ejecución de los proyectos no fue la planificada, debido a un sin número de factores, internos como externos, que impactaron de forma negativa en la ejecución de diversos proyectos.

\section{Rentabilidad}

Se tomará como ejemplo un proyecto para el servicio público de energía eléctrica, enfocado al mejoramiento de la calidad del servicio eléctrico, mediante el reemplazo, reubicación, readecuación, repotenciación, reconfiguración de líneas, equipos y otros componentes del sistema eléctrico, iniciando su ejecución en el mes de enero del año 2019 y culminando en el mes de marzo del 2020, denominado "Repotenciación y Reconfiguración de un alimentador".

El alcance de este proyecto fue el de mejorar la calidad del servicio eléctrico debido a que el alimentador había cumplido su vida útil, con el fin de cubrir la demanda actual y futura y así evitar las salidas del alimentador (cortes de servicio), mejorando la confiabilidad del sistema eléctrico.

El proyecto apuntó al objetivo estratégico: Incrementar los niveles de eficiencia de distribución eléctrica y alumbrado público, ya que permitió mejorar la calidad y eficiencia del servicio, con una inversión de $\$ 725.850,00$ y un plazo de ejecución de 180 días (planificación inicial).

Se consideraron los siguientes parámetros para el cálculo de la rentabilidad (establecidos por el MERNNR): 
- Llevar a cabo proyectos de telemetría (lectura inteligente).

- Precio promedio de compra de energía: 0.0370

- Precio medio de venta al cliente: 0.0945

- Costo de O\&M (operación y mantenimiento): $6 \%$

- Tasa de descuento: $10,80 \%$

Parámetros establecidos por CNEL:

- Tasa de crecimiento de demanda: $4 \%$

- Factor de carga: 0,64

- Tasa de inflación (costo de O\&M): $2 \%$

Para su rentabilidad, se consideran 15 años del período de vida útil del alimentador, y se toma en consideración la inversión inicial, el margen neto por venta de energía y los costos de O\&M (operación y mantenimiento) que este proyecto implicaría durante los 15 años.

Al ser una organización dedicada al servicio de la comunidad, todo proyecto va a generar un beneficio social. En este proyecto, el mantener el alimentador existente le permitía generar a la empresa una distribución de $12.465 \mathrm{MWh}$ anual, sin embargo, con esta repotenciación y reconfiguración, a la empresa distribuidora le permitió generar mayor MWh, desde 12.465 hasta 26.132, entregando mejor calidad de servicio y permitiendo la expansión del mismo a más residentes.

Durante la gestión del proyecto se observaron algunos inconvenientes durante su ejecución, a pesar de que el mismo culminó cumpliendo su objetivo técnico:

- El proceso de obra y de fiscalización fueron adjudicados en la segunda publicación de los mismos, lo cual implicó atrasos de 60 días adicionales a su plazo planificado de contratación.

- Incumplimiento por parte de contratistas.

- Incremento del presupuesto debido a que los precios unitarios variaron de año a año.

- Atrasos en la importación de bienes (responsabilidad del contratista).

- El proyecto cumplió con su alcance, mas no en tiempo y costo.

- Demora en la gestión legal para emitir las respectivas resoluciones de incumplimientos.

- Imposición de multas debido al atraso en la firma de actas de entrega recepción definitivas entre el contratistas y administrador de contrato.

- La obra se culminó 270 días después del plazo de ejecución. 
El proyecto fue ejecutado mediante dos procesos de contratación, la obra y su respectiva fiscalización. Mediante la contratación y ejecución de estos procesos, se logró culminar el proyecto.

\section{Impacto Social}

La comunidad del sector en el que se llevó a cabo este proyecto pudo incrementar sus niveles comerciales, colocando talleres, locales comerciales y fábricas lo que, debido a la baja capacidad del alimentador anterior y a sus fallas técnicas, no era posible.

Esto permitió dar nuevas oportunidades para su desarrollo social y económico, ya que ahora cuentan con un nuevo abastecimiento de energía en mejores condiciones. Así también se pudo otorgar de un mejor servicio en el alumbrado público.

Ampliando un poco esta temática debe observarse que el sector eléctrico en general es estratégico y que necesita todo tipo de empleados desde administrativos, de primera línea, técnicos hasta gerenciales por lo que brinda empleos directos a las familias.

Por otro lado, al estar mayores zonas electrificadas y al evitar las pérdidas negras por conexiones clandestinas los negocios aumentan, las familias logran tener acceso a la electricidad lo que le permite estudiar, cocinar, adquirir electrodomésticos y ayuda a cubrir las necesidades insatisfechas, estos serían beneficiarios indirectos.

Dado lo anterior el realizar de forma técnica, profesional y recurrente permite a las empresas que compiten en el sector como al estado tener una clara idea del impacto no solo social sino económico que conlleva que estos proyectos se cristalicen pese a la cantidad de recursos que se usan de una manera planificada y estratégica.

\section{DISCUSIÓN}

El análisis realizado nos permite identificar que para poder cumplir con una adecuada planificación estratégica se deben definir objetivos, y para poderlos realizar se plantean acciones, dentro de las cuales se identifican diversas tácticas que derivan en ejecuciones de varios proyectos, que, de realizarlo de forma correcta, permitirá generar valor a la organización.

Así también, mediante la observación efectuada a la operatividad de la organización, se identificó que no existe un trabajo en equipo que permita que sus labores en conjunto conlleven al cumplimiento de los diversos objetivos estratégicos planteados en su planificación, tal es el resultado que las acciones que efectúan son correctivas, esto implica que su labor no esté alineada a la consecución de las metas. 
Las ejecuciones presupuestarias de los proyectos de inversión es significativamente baja, reflejando una deficiencia en las diversas gestiones que impactan en la gestión de los proyectos o en sus procedimientos inmersos, como por ejemplo cuellos de botella, provocando que los recursos no empleados no aprovechen costos de oportunidad, esto a pesar de que los proyectos si permiten cumplir con los indicadores técnicos, como por ejemplo los índices de pérdidas técnicas, cobertura del servicio y expansión del servicio eléctrico.

A pesar de que existen procedimientos y procesos ya establecidos, estos no son cumplidos en su totalidad, ni tampoco se efectúa un control de calidad que permita identificar mejoras para los mismos. Estos inconvenientes impactan en los tiempos de ejecución, provocando que se tomen acciones para solventar inconvenientes presentados en una situación actual sin que se contemple una mejora continua que alimente las lecciones aprendidas a aplicar en situaciones similares a futuros proyectos, como lo describe la teoría de gestión de procesos.

Se considera conocer de teorías en gestión de proyectos, con el fin de evitar fracaso en los mismos. Con base en el análisis efectuado, se pudo evidenciar que previo a la ejecución de un proyecto, no se identifican los posibles riesgos que vayan a afectar su gestión, tampoco se plantea un plan de respuesta en caso materializarse, que permita mitigar o eliminar los impactos que puedan causar.

Se debe considerar dentro de los procedimientos establecidos para una planificación estratégica, los mecanismos para identificar desviaciones que se presenten en los proyectos, es decir cuando no se está cumpliendo con el tiempo, costo, ni alcance, y que permitan tomar medidas correctivas sin afectar a la gestión interna. También se identificó que no cuentan con herramientas automatizadas que permitan facilitar el seguimiento y control a un extenso y diverso portafolio de proyectos.

Como toda empresa gubernamental, uno de los factores que influye en la gestión de proyectos de inversión son los constantes cambios administrativos, a niveles jerárquicos, administrativos y operacionales, impactando en el tiempo ya que sus aprobaciones y flujo de información tomarán más tiempo de lo planificado, esto debido al tiempo de adaptación y conocimiento general de la situación para aquellos nuevos colaboradores de la institución, así también influyen de forma significativa en el cumplimiento de metas establecidas en los objetivos estratégicos.

De acuerdo a lo analizado se plantea la siguiente matriz que servirá para futuras investigaciones. 
Tabla 2

Factores críticos

\begin{tabular}{l} 
OBJETIVO \\
\hline Analizar la gestión de procesos \\
de los proyectos de inversión y \\
su relación en la planificación \\
estratégica en el sector \\
eléctrico ecuatoriano
\end{tabular}

eléctrico ecuatoriano

Realizar una revisión teórica de las principales teorías de la administración estratégica, la gestión de procesos, gestión de proyectos y plan de dirección de proyectos

Realizar un diagnóstico sobre la planificación estratégica existente en relación a la ejecución los proyectos de inversión, además de evaluar el estado actual de los procesos asociados a los proyectos de inversión.

Analizar de forma crítica los factores que se relacionan a los proyectos de inversión
No se aplican las teorías de gestión de proyectos.

No se efectúa un análisis a nivel de proyectos y portafolio de forma integral, de tal manera que los resultados obtenidos en conjunto permitan agregar valor a la empresa

No se efectúa un plan integral de gestión de proyectos
RECOMENDACIÓN

Se debe realizar una auditoría de procesos, y de ser el caso, aplicar reingenierías a los mismos, permitiendo definir una adecuada planificación para cumplir con las metas, empleando a su vez herramientas básicas que contribuyan a una buena gestión de proyectos de forma eficiente $y$ obteniendo los resultados deseados a favor de la comunidad.

Se debe identificar debilidades en las áreas gestoras de proyectos, así como también impartir las capacitaciones sobre sus teorías de gestión, que permita administrar, orientar y diseñar todo esfuerzo dentro de un proyecto, de principio a fin.

Se debe efectuar un análisis de forma integral a nivel de programas y portafolio, que conlleva a la identificación de los canales o medios para poder cumplir con las metas establecidas, así como también efectuar controles permanentes a la planificación estratégica.

Aplicar metodologías que permitan mejorar la gestión de forma interna en la PMO o Dirección de Proyectos, y que a su vez esta vaya encaminada a ejercer funciones de una PMO de dirección y control, alcanzando la madurez necesaria para gestionar proyectos de calidad y con éxito.

Fuente: Elaboración propia

\section{CONCLUSIONES}

Al analizar la gestión de procesos de los proyectos de inversión y su relación en la planificación estratégica en el sector eléctrico ecuatoriano, se concluye que la planificación estratégica determina los objetivos hacia los cuales apunta la organización o entidad pública, y de esta se desprenden las estrategias o acciones que se deben realizar para poder llegar a este cumplimiento. 
Sin embargo, una de los más importantes factores que afecta a la ejecución de los proyectos, los cuales aportan al crecimiento económico y social de las comunidades, es la restricción de los recursos disponibles para llevar a cabo sus ejecuciones, de tal forma que limita abarcar las necesidades o demandas, es por ello que se considera necesario el poder acceder a más aportaciones de recursos, que provengan de organizaciones externas como inversión, y que con ello se prioricen las iniciativas de proyectos que son presentadas por las empresas distribuidoras, que permita en primera instancia dotar de servicio a lugares con demandas más acentuadas y en donde no se tenga acceso al servicio eléctrico, así como también reforzar subestaciones o líneas de subtransmisión para suplir del servicio a los mismos, esto permitirá que los indicadores de expansión del servicio eléctrico incrementen, así como también los indicadores de viviendas nuevas atendidas o con mejoras.

En cuanto a las bondades que nos dan las diversas teorías, sean estas de administración, procesos, estratégica o proyectos, se evidencia que existen debilidades en las gestiones internas que no permiten soportar al $100 \%$ todas las gestiones de apoyo existentes.

El personal que ejerce funciones de gestión de proyectos, también tienen a cargo funciones operativas, que no permite centrar sus tareas en todas las aristas que son ejecutadas dentro de una obra, sin que pueda alertar de forma proactiva aquellas desviaciones que se presentan en las fases o etapas de un proyecto, para ello es necesario que el área de apoyo o área gestora de proyectos se convierta en una Oficina de Proyectos Proyectizada (orientada a proyectos), en donde las funciones sean enfocadas al liderazgo, control y responsabilidad únicamente de las obras eléctricas, considerando que la cantidad de obras puede incrementar a números incontrolables, permitiendo aplicar metodologías en gestión de proyectos y que a su vez gestionen las expectativas de la gente relacionada 0 afectada con los mismos, sean estas internas o externas.

Uno de los beneficios que permitirá otorgar la correcta gestión de proyectos, es poder cumplir con los compromisos dados a la comunidad, permitiendo optimizar recursos que sin duda alguna podrán ser empleados en más requerimientos para cubrir la demanda e incrementar la recaudación, lo que puede impactar en mayores inversiones.

Al realizar un diagnóstico sobre la planificación estratégica existente en relación a la ejecución los proyectos, además de evaluar la situación actual de los procesos vinculados a los mismos, se identificó que no existe un proceso claramente definido de control y seguimiento que permita generar entregables (resultados o productos de proyectos) con calidad, para lo cual se recomienda emprender auditorías en los diversos procesos empleados, identificando errores y aplicando mejoras continuas, conocidas también como buenas prácticas. 
La madurez en la gestión de proyectos, sin duda alguna permitirá extender el portafolio de los mismos y suplir necesidades emergentes en sectores estratégicos, como es el caso actual de la electrificación de la industria camaronera, generando una acuacultura eficiente y sostenible, permitiendo incrementar el proceso de aireación eléctrica, tener un control automatizado de las fincas camaronera, así como de un bombeo eficiente.

Es necesario poder definir al inicio de todo proyecto el alcance, que permita determinar de forma sencilla y clara todos los objetivos que se pretenden obtener con la ejecución del mismo, siendo medible, alcanzable, realista y con un tiempo definido, qué es lo que está considerado dentro del proyecto, es decir qué se debe hacer, cuándo se lo debe hacer, cómo se debe hacer y quienes lo deben hacer, identificando posibles riesgos y todo costo que implicaría en caso de que estos se lleguen a materializar, así como también definir un plan de comunicación y cómo este debe manejarse durante la ejecución de un proyecto.

Se considera necesario recalcar que la gestión de proyectos es un proceso integrador, que define: planes de alcance, costo, cronograma, recursos, gestión de comunicaciones, calidad, riesgos, interesados y las adquisiciones a efectuar, definidas por el PMI y consideradas dentro de las buenas prácticas para contribuir a las posibilidades de éxito de una amplia gama de proyectos y de ello desprender mejoras continuas aplicables a proyectos de diversas índoles.

\section{REFERENCIAS BIBLIOGRÁFICAS}

Asociación española para la calidad. (2019). Asociación española para la calidad. Obtenido de https://www.aec.es/web/guest/centro-conocimiento/gestion-por-procesos

Concepto Definición. (11 de septiembre de 2019). Concepto Definición. Obtenido de Concepto Definición: https://conceptodefinicion.de/proceso/

Edinburgh Business School. (2014). Gestión de proyectos. Edimburgo: Heriot-Watt University.

ESAN. (27 de octubre de 2016). CONEXION ESAN. Obtenido de CONEXION ESAN: https://www.esan.edu.pe/apuntes-empresariales/2016/10/que-es-la-administracionestrategica/

IDDEO Consulting. (s.f.). La Gestión por Procesos. Zaragoza.

Juan José Miranda Miranda. (2002). Gestión de Proyectos. Bogotá: MM Editores.

Ministerio de Energía y Recursos Naturales No Renovables. (2019). Plan Maestro de Electrificación 2018 - 2027. Quito.

Ochoa, J. (2018). Metodología para la gestión de proyectos y portafolios en una empresa eléctrica. Guayaquil. 
PMI Madrid Spain Chapter. (06 de diciembre de 2018). PMI Madrid Spain Chapter. Obtenido de PMI Madrid Spain Chapter: https://pmi-mad.org/index.php/socios/articulosdireccion-proyectos/1909-la-importancia-de-la-buena-gestion-de-proyectos-en-lasorganizaciones

Project Management Institute. (2017). GUÍA DE LOS FUNDAMENTOS PARA LA DIRECCIÓN DE PROYECTOS - GUÍA DEL PMBOK. Pennsylvania: Project Management Institute, Inc.

Project Management Institute. (2017). PMBOK.

Revista Ciencias Estratégicas. (2016). Análisis de la problemática de la gestión: estudio en el contexto empresarial colombiano. Revista Ciencias Estratégicas, 135.

Vanegas, E. A. (s.f.). Diseño de la PMO para Oleoducto Central S.A. Diseño de la PMO para Oleoducto Central S.A. Universidad EAFIT, Bogotá.

Zacarías Torres Hernández. (2014). Administración Estratégica. México: Patria. 